\title{
Appendix
}


List of sections of AMNH 32949h, embryo 1 in rostral to caudal (RC) sequence correlated with previous numbering schemes

RC Packet Ink Slide Comments ${ }^{5}$

number number number size

RC Packet Ink Slide Comments

number number number size

$\mathrm{RC} 1$

A6 549

1

lantern

$\mathrm{RC} 2$

A6 548

2

$\mathrm{RC} 3$

A6 547

3

RC4

A6 546

4

RC5

A6 544

5

RC6

A6 543

6

RC7

A6 542

7

RC8

A6 541

8 
RC9

A6 539

lantern

RC10

A6 538

10

RC 11

A6 537

11

$\mathrm{RC} 12$

A6 536

12

RC 13

A6 534

13

$\mathrm{RC} 14$

A6 532

14

RC 15

A6 531

15

lantern

RC16

A6 530

16

lantern

RC 17

A6 528

17

RC18

A6 527

18 
RC19

A6 526

19

RC20

A6 525

20

RC21

A6 524

21

lantern

RC22

A6 523

22

$\mathrm{RC} 23$

A6 522

23

RC24

A6 521

24

RC25

A6 519

25

RC26

A6 518

26

RC27

A6 $5 \mathrm{I} 7$

27

RC28

A6 516

28

RC 29

A6 515

29

RC30

A6 514

30

RC31 
A6 513

31

RC 32

A6 512

32

RC33

A6 511

33

RC34

A6 510

34

RC35

A6 509

35

RC36

A6 50S

36

RC37

A6 507

37

RC38

A6 506

38

RC39

A6 505

39

RC40

A6 505

40

RC41

A6 504

41

RC42

A6 502

42

RC43

A6 501

43

RC44

A6 500

44

RC45

A5 499

45 
lantern

lantern

lantern

lantern Duplicate No. A6 505

lantern

The sections were cut, stained and mounted in the laboratory of M. Lagios. They were placed in rostral to caudal (RC) order and double checked before paper labels with RC numbers were placed on the slides.

This is an obsolete set of numbers written on each slide by the original processors; we refer to them as 'Packet Numbers'; they are typed, and consist of a packet letter followed by the number. Many of these numbers were inadvertently duplicated during transcription, as indicated under the 'Comments' column.

This is an obsolete set of numbers written in ink and thus referred to as 'Ink Numbers'. They presumably represent an earlier attempt to correctly order the slides.

If not indicated in this column, then the section was mounted on a standard 2"x3" (51 mmx76 mm) slide. Some of the sections were mounted on $3.25 "$ x 4" $(83 \mathrm{~mm} \times 102 \mathrm{~mm})$ lantern slides. The two series of slides are stored in separate boxes.

Many of the sections were mounted upside down. This was corrected by placing red paper dots in a standard corner on every slide. When holding a slide so that the red dot is in its lower right corner, dorsal is to the top of the slide and the anatomical left side of the specimen is towards the right. 
707070 TO 707070 ?d 7373 po 70 pO 73 jo $70 / O 7070$ po /O p3 p3 jo ?3 70 To TO 70 TO TO TO TO 7070707070707070707070 TO $7070707 \&$ TO TO TO 707070 0000000000000000000000000000000000000000000000000000000

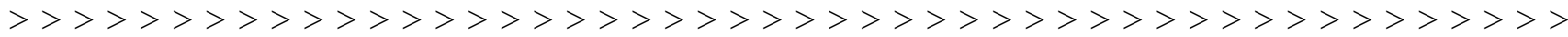
$4 \wedge$ 4- 4- 4- $4 \wedge$ 4- 4-

4.i.titt£t(yi'yiLnO

$U x \mathrm{~L} / \mathrm{t}$

$>$

$>$

$>$

$>$

*

t/l

$\mathrm{L} / \mathrm{i}$

$\mathrm{Gi}$

ri

4-

cr

*

$\mathrm{t} / \mathrm{l}$

$\wedge_{\mathrm{n}}$

lo

re

C/i

${ }_{-0}^{-0 N}$

H $\quad 0 \ll 5 \quad 00>$ J

£3. W

D 
I O

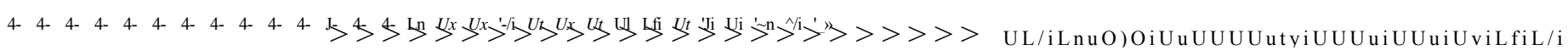
$\mathrm{s}^{\wedge} \mathrm{s} \mathrm{s} \mathrm{S}$ s S S S s tf $\mathrm{g}^{\wedge} \operatorname{gSS} \sin 11^{\wedge} \wedge \operatorname{s} 1111 \mathrm{Sg} \lg i 1 \mathrm{SSSSSgSg} \operatorname{siSgSgggSS}$

g- $H$

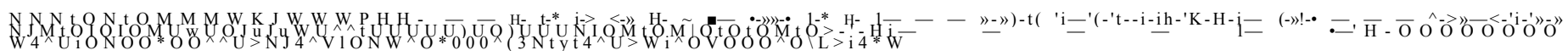
$\mathrm{I} £$

$£ 2-\mathrm{Cft}$

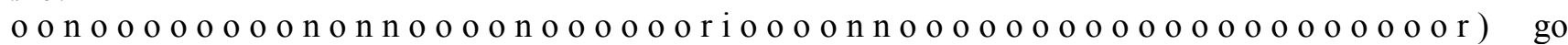

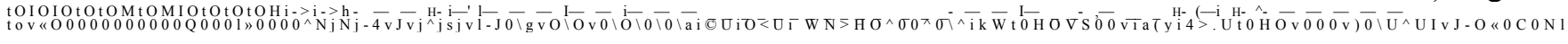
$>>>>>>>$

4 - J - 4 - 4 - 4 - 4 - - U 4

$>>>>>>>>>>$

4 - 4 - 4 - 4 - 4 - 4 - 4 - $-4-4$ - X - 4 - 4 -

$>>>>>>>>>>>>>>>>>>>>$

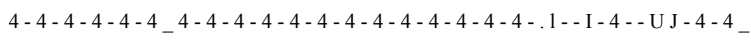

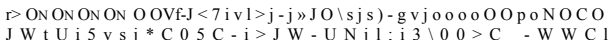

$>>>>>>$

4^. 4^ 4- 4- 4- 4- 4-

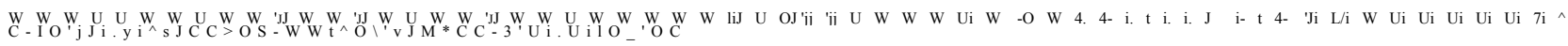




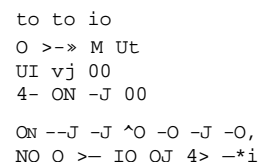

\section{$\S \mathrm{SSB}$}

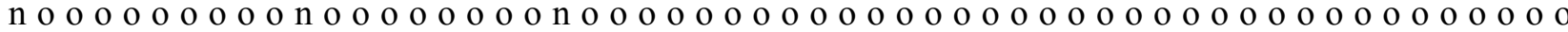

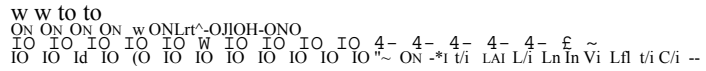

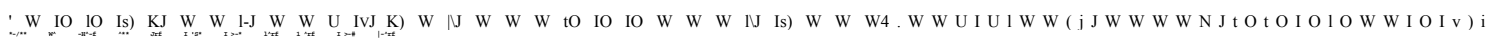

W to

C-toe-0'0CO-0.ON-n

U) $\mathrm{IO}-5$

CO $-J$ ON Ui $4-$

$4-$ - jJlO-COO5-OONW4-

LJ OJ

to to to Io to to to to to to to to Io to to to to to to Io to to Io to to to

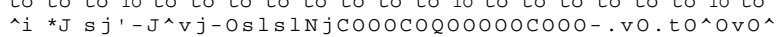

C 0 IO 0 J 4 -

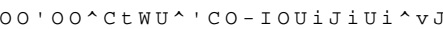

$t$
$v$

G $0<0$ O $01-\mathrm{U} 6 \mathrm{U} 0$

W W OJ OJ W W UJ -J CO No O >-- to $\mathrm{W}$

LtJ OJ $\leadsto$ UJM H IN) (O

W Ui U U U U to to to to to to $U>4-$ Wi ON-O: CO

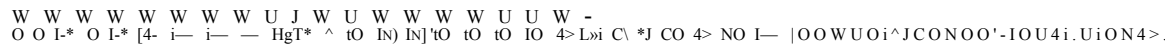




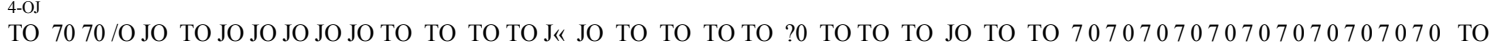
$7070707070^{\wedge} 7070707070$ TO TO ^? 3

o o o noo noo o o o n noo o o o o o o o o o o o o o o o o o o noo o o o o o

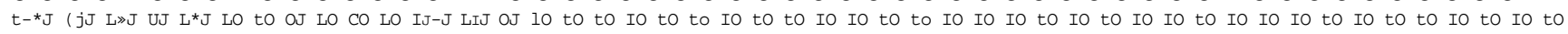

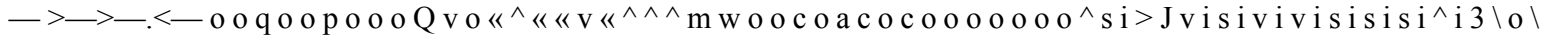

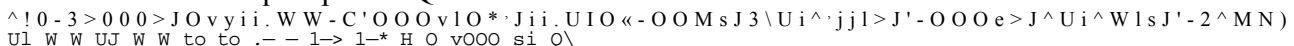

$>>>>>>>>>>>>>>>>>>>>>>>>>>>>>>>>>>>>>>>>>>P$ U U U W V U

to to to to to to to to to to to to to to to to 10 to 10 to to to to to to to to

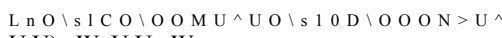

UU) W U U W

W W OJ Ui W W

$01 \mathrm{co}$

$\mathrm{U} 10 \mathrm{U} \mathrm{u}>$

Co CO CO CO

10 to to

W 'JJ W U W W W W U W W 'jJ W U W w

tofouto to to to $i$. $7 i$ si $M » C$

$1-$ IO
$\mathrm{W}<3>$

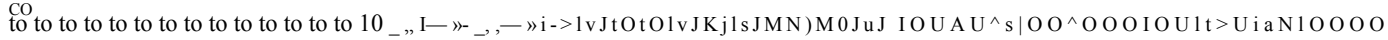

to to to to to to

i JW t l $/$ i $\wedge$

to to to to to to to $\mathrm{C} / \mathrm{i} / \mathrm{C} / \mathrm{Lft}$ ONONONON

to to to to to ONOvOr Or ON

$\mathrm{L} / \mathrm{ON}-\mathrm{J}$ CO NO

to to to to to to to to to to 10 to to to to to to to to to to to 1010 to to

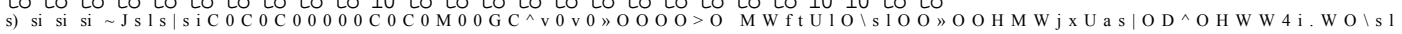


o o o o o o o o o o o o o n o o o o o o o o o no

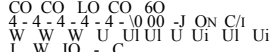

70
7

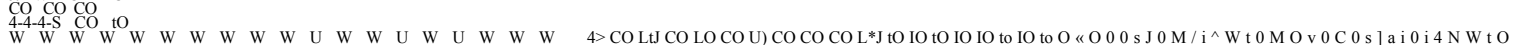

$7070^{\wedge} \wedge 707 \mathrm{O} ? 3^{\wedge} 73$ ? O 70707 a $7 \mathrm{O}^{\wedge} 707^{\wedge}$ ? $\mathrm{O}^{\wedge}$

O O O O O O O $\mathrm{n}_{\mathrm{W}}^{\mathrm{n}} \mathrm{O}$ O

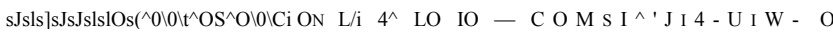

$\mathbf{i}^{\circ}$

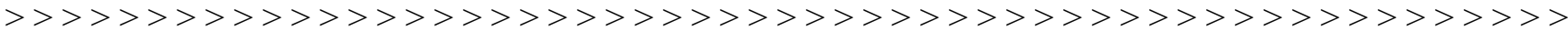

to 10 to to to to to to to 10 to to to 10 to to to to to to

10 to 10 to

Io Io to Io to Io to Io to Io Io to to Io Io to to Io to Io to Io to Io co Lo co Lo ct.)

CO CO CO CO $\mathrm{CO}$ CO CO $\mathrm{CO}$ ON OS
CO CO

ON C/I ON ON ON ON ON CN ON ON ---J

Osl-MUiAWslftHOO

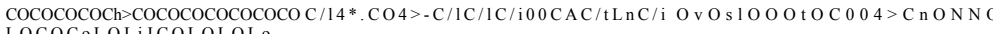

LOCOCOLOLIJCOLOLOL

CQOOCONDNO^ONONOVONONOCOCO^.^

COCOCOCOCOCOCOCOCOCOCOCOCOCOCOCOCOIONJ^EOtO

i*s J OOQ Q $>$ \&

sj.

to $\mathrm{COC} / \mathrm{f}$

$¥$

B

a 
737373737318737tW73737373737373737373iaW73737373W

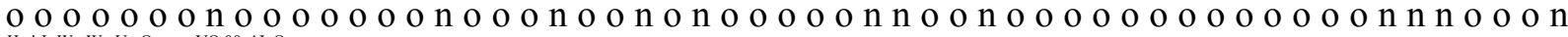
U. W W U vo vp vo 00 ^J Qs

$>>>>$

4a. $4>4 * 4 * 4^{*}, 4^{*}, 4^{*}$

$\mathrm{W} \mathrm{W} \mathrm{W}$ N) $\mathrm{W}$ W !U Is) IO N) IO to $-\ldots$

$>>>>>>>>>$

$>>>;>;>;>>;>>>>>>;>>;>>;>>;>>>>>>>;>;>>>. ;>>;>>$

ig $w$ to io $w \mathrm{w}-\mathrm{w} w$ io io $\mathrm{w}$ io $\mathrm{w} w \mathrm{w}$ u io io $\mathrm{w} m$ io io $\mathrm{w}$ io $\mathrm{w}$ io [J to io $\mathrm{w}$

$3 \&$

IO

$\mathrm{o}^{*} \mathrm{a}$

1>J $4^{*}-$ Ln $\mathrm{i} \rightarrow \gg 0$

WW U U

$\gg) \mathrm{U} U \mathrm{U}$

4a.4i.4i.

tS $§ S 33 m S ; 33 £ \S$

$00 \quad \mathrm{CC} \quad \mathrm{CO}$ IO IO IO $\mathrm{U}>\mathrm{U}$ ) U) OJ $-\mathrm{J}$ OJ> Ln

$\mathrm{o}$ - to -o co o c; - to OJ ui -i- — -

- I O U I U A ^ - O OOOO

"SSSS

333

a

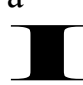




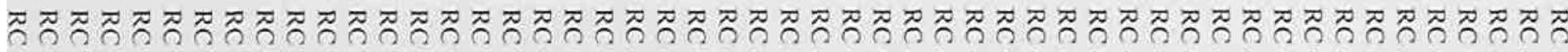

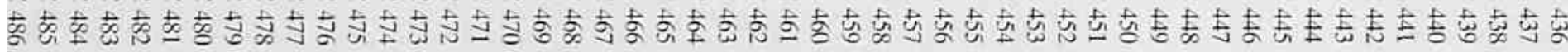

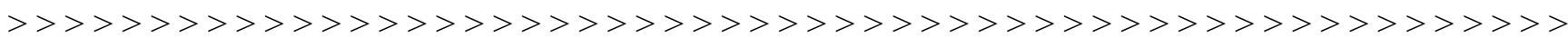
$£ £$

IO IO IO IO $\mathrm{k}$

IO IO IO $\mathrm{U}$ W $\mathrm{W} \wedge$ IO $1>\mathrm{J}$ W W

W U ) i $-4-4 \wedge 4$ i - W C O W

iiLflUl^vyiUiL/iLflWUiLn

AwauwaoN^${ }^{\wedge} \mathrm{ft}^{\prime} \mathrm{ON}$

3 "T $3 \mathrm{C}$ ea $3 . \quad 2$.

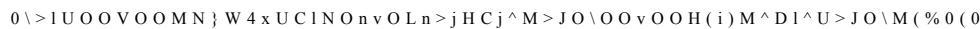

"9 re3 a.

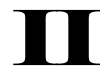

I

$\mathbf{I}$

I

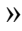


$\begin{array}{ll}3 & \\ 3 & 3\end{array}$

D

0

H.

*2-

"H.

re 
$l \mathrm{w}$

TO 70 TO 70737370707070 TO 70

o o o o n o o o o o o

\section{$\&$ S S ;}

\section{$51 £ 3 \S$}

707^70707070707070707373707070737070 n o o o n o o o o o o o o o o o

L/ / L / ILALALALALALALALALALALALALALALALA 4^ 4> La Lo La La La La La La La La to to to to to to 70707373737073737070

O O O O D O O O O П O O O O O O O O O O O O O O

LA LA LA LA LA LA LA LA LA LA LA LA LA LA LA LA LA $4 \wedge$. $4^{\wedge}-$

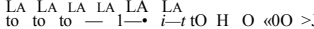

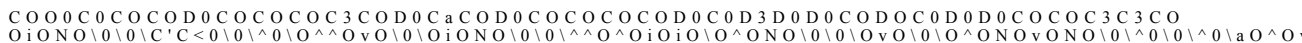

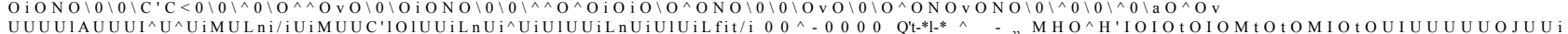

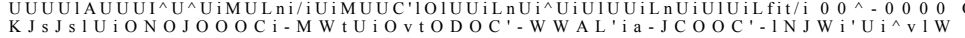

L A L A L A L A t/ / L A L A L A L A L A L A 
$\mathrm{WJi} * .4 \mathrm{v} 4 . \mathrm{ft}^{\wedge} 4.4-\mathrm{Ui}$

$\mathrm{DO}>>>>>$

LA LA K $-4-\mathrm{L} / \mathrm{O}$ OO

\section{IS}

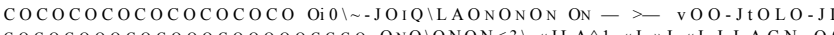

GOCOCOOOCOCOOOOOCOOOOOCOOOOOCOOOOOOOOOCO -J -O-OCOCOCOCOQOOOCOCOCOCONONONONONOONO -O O0 NO O ba(OLA 4 S-LAON-OCONOOK$\mathrm{COL}$ a 4 L $\mathrm{L} / \mathrm{iON}$
$\mathrm{CO}$ CO NOvo VO O

$\stackrel{\mathrm{vO}}{000 \mathrm{VO}} \mathrm{VO} \rightarrow$ to $\mathrm{C} 5$

NO NO LOQ Q VO $4^{\wedge}$ LA VO

$\mathrm{I}<\mathrm{ls} 2$

$\mathrm{N}$ ST.

$\mathrm{O}$
$\mathrm{C}$
13

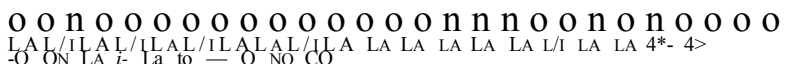

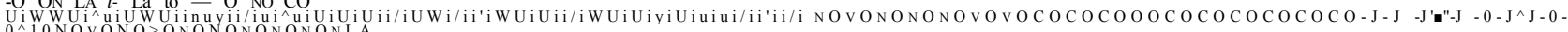

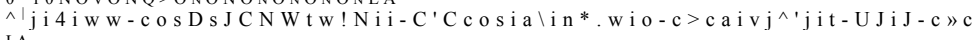

LA

$\dot{1}$

COCOCOCOCODODOCOCOCSCOOOCOCOCODOCOOOCOOOCOCOCOCDCODOCOOODOOT

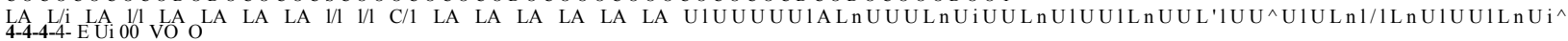

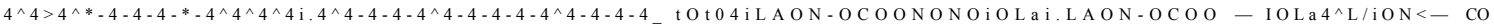

a $>-O$ a 0 .

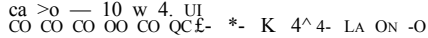

$\S 11111^{a}$ - 


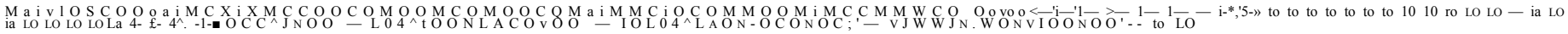
$50 ? 0$ ja

ON ON ON

\section{SSls}

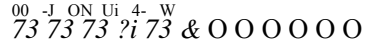

7373737373737373737373737373737373737373737373737373

o o o o o o o o no o o o o o o no o n o o

ONONONONONONONONONONONONONON

$\mathrm{U} W \mathrm{~W}$ UI IO IO IO IO $\mathrm{W} W$ IO $\mathrm{W}$ IJ $\mathrm{W}$

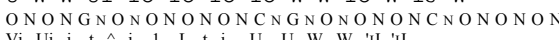

73737373737373737373737373737373

o o o o o o o n o o o o o o o o

ON

ON ON

o *o 00 -j ON u»

$0>\mathrm{ONONO} \backslash \mathrm{QNQNONONQ}$

3

CdCOCOCOCOCOCOCOCOCOcdCOCOCdCOCOCOCOCOCO uiuiuiuiuiuiuiuiuiuiuiuiuiuiuiuiuiuiuiui

$4-4-4-\mathrm{W}$
$\left.\mathrm{H} \mathrm{W}_{\mathrm{W}} \mathrm{U}\right)$

cococococococococococococococococococococococococccococo

i. i. $4 \wedge$ 4- 4- J- J- $\wedge$ t i- $4-$ A- 'Ji Ui Ui Ui Ui Ui in $w$ Ui U Ui U W Ui 7 i Ui

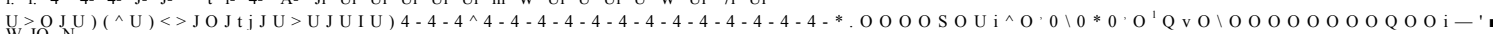

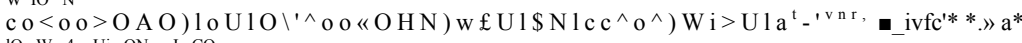

IO W $4-\mathrm{Ui}$ ON $-\mathrm{J}$ CO

CO CO COUi Ui U

\section{$\circ$ 4? JS SIS! S 1}

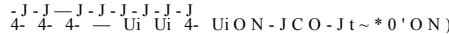

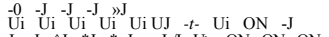

0 《o o h to 01

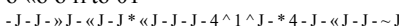

$\mathrm{mo} \backslash-\mathrm{J} 00$

$-* \mathrm{~J}-\mathrm{J}-\mathrm{J}-\mathrm{J}-\mathrm{J}-\mathrm{J}-* \mathrm{~J}-\mathrm{J}-\ll \mathrm{J}-\mathrm{J}$

OOOOCOOOQOOOOOOOOOOO,ONO,OVO

$\mathrm{C}-\mathrm{WW} 4 \mathrm{iUiCs}-\mathrm{JCOOO}-\mathrm{I-J} \mathrm{LO}$ 
noo o o n o n o o o o o o o n o o o o o o o onnorjoorjoooooonoooooonoooono คิ人 $\mathrm{O}$

\section{$222 \operatorname{gggggggggggg} 22 \mathrm{~g} 22222 \mathrm{~g} 22222222 \mathrm{~g}$}

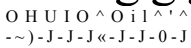

H M - I - O I -

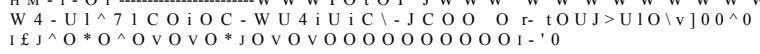

$>$

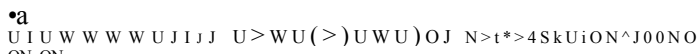

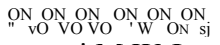

o o u i M W J > u i a $>\wedge$ o o H OH i ow

4^ 4^- Ui U!HOI UUUIUIUIUIUIUIONONONONCTVONQN

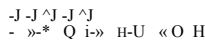

co $\operatorname{coco}$

\section{$\operatorname{SSS}^{\wedge} \mathbf{S}$}

COCOCOCOCOCOCOCOCOCOCOCOCOCOCD

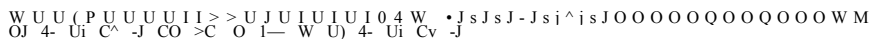

D

13

Packet number ${ }^{1}$

Ink $\quad$ Sliclelnumber size ${ }^{4}$

presumed presumed 
presumed presumed presumed presumed presumed presumed presumed

662 presumed presumed presumed presumed presumed presumed presumed presumed presumed

661 presumed presumed presumed presumed presumed presumed presumed presumed presumed

660 presumed presumed presumed presumed presumed presumed presumed presumed

659 presumed presumed presumed presumed presumed presumed presumed presumed presumed

658 presumed presumed presumed presumed presumed presumed

section section section section section section section section section B3 270 section section section section section section section section section B3

260 section section section section section section section section section B3 240 section section section section section section section section B3 250

section section section section section section section section section B3 230 section section section section section section

ost during ost during ost during ost during ost during ost during ost during ost during ost during

lantern ost during ost during ost during ost during ost during ost during ost during ost during ost during

lantern ost during ost during ost during ost during ost during ost during ost during ost during ost during

lantern ost during ost during ost during ost during ost during ost during ost during ost during

lantern ost during ost during ost during ost during ost during ost during ost during ost during ost during

lantern ost during ost during ost during ost during ost during ost during

$\mathrm{RC}$

Packet Ink

Slide

Comments

number $^{1}$

number ${ }^{2}$ number

size

RC707

B4

RC708

B4

$330 \quad 689$

RC 709

B4 
RC 700

$\begin{array}{ll}\text { B4 } & \\ 328 & 687\end{array}$

RC 711

B4

$327 \quad 686$

RC712

B4

$326 \quad 685$

RC 713

B4

$324 \quad 682$

RC714

B4

$325 \quad 684$

RC715

B4

$323 \quad 681$ 
RC716

B4

$322 \quad 680$

RC717

B4

$321 \quad 679$

RC718

B4

$320 \quad 678$

RC719

B4

$319 \quad 677$

RC720

B4

$318 \quad 676$

RC721

B4

$317 \quad 675$ 
RC722

B4

$316 \quad 674$

RC 723

B4

$315 \quad 673$

RC724

B4

$314 \quad 672$

RC725

B4

$313 \cdot 671$

RC726

B4

$310 \quad 668$

RC 727

B4

$312 \quad 670$ 
RC 728

B4

$311 \quad 669$

lantern

RC729

B4

$325 \quad 683$

lantern

B4 325

RC730

B4

$303 \quad 667$

lantern

RC731

B3

? 666

RC732

section presumed

ost during

processing

RC733

section presumed

ost during

processing

RC734

section presumed

ost during

processing

RC735 
section presumed

ost during

processing

\section{RC736}

section presumed ost during

processing

RC737

section presumed ost during processing

\section{RC73S}

section presumed

ost during

processing

\section{RC739}

section presumed

ost during

processing

RC740

section presumed

ost during

processing

RC741

B3

$298 \quad 665$

lantern

\section{RC742}

section presumed ost during

processing

\section{RC743}


section presumed

ost during

processing

RC744

section presumed

ost during

processing

RC745

sectieBrbresumed

ost during

processing

\section{RC746}

sectiHforesumed

ost during

processing

RC747

secti^${ }^{\wedge}$ Spresumed

ost during

processing

RC748

section presumed

ost during

processing

\section{RC749}

section Disjoined

ost during

processing

\section{RC750}

section presumed

ost during

processing

RC751

B3 
$289 \quad 664$

lantern

\section{RC752}

section presumed

ost during

processing

\section{RC753}

section presumed ost during processing

\section{$\mathrm{RC} 754$}

section presumed ost during processing

\section{RC 755}

section presumed ost during

processing

\section{RC756}

section presumed

ost during

processing

\section{RC757}

section presumed ost during

processing

\section{$\mathrm{RC} 758$}

section presumed ost during processing

\section{RC759}

section presumed ost during 
processing

RC760

section presumed

ost during

processing

RC761

B3

$280 \quad 663$

lantern

$\mathrm{RC}$

number

RC762 RC763

RC764 RC765 RC766 RC767 RC768 RC769 RC770 RC771 RC 772 RC773 RC774 RC 775 RC 776 RC777 RC 778 RC779 RC 780 RC7S1 RC7S2 RC783 RC7S4 RC785 RC7S6 RC7S7 RC788 RC789 RC790 RC791 RC792 RC793 RC794 RC795 RC796 RC797 RC798 RC 799 RC800 RC801 RC802 RC803 RC804 RC805 RC806 RC807 RC808 RC809 RC810 RC811 RC812 RCS13 RC814 RCS15 RC816

Comments

processing processing processing processing processing processing g processing processing processing

processing processing processing processing processing processing processing processing processing

processing processing processing processing processing processing processing processing processing

processing processing processing processing processing processing processing processing

processing processing processing processing processing processing processing processing processing

processing processing processing processing processing processing

69

Comment?

Slide 


\section{processing processing processing}

processing processing processing processing processing processing processing processing processing

processing processing processing processing processing processing processing processing processing

lost during lost during lost during

lantern lost during lost during lost during lost during lost during lost during lost during lost during lost during lantern lost during lost during lost during lost during lost during lost during lost during lost during lost during lantern

lantern Duplicate No. B2 198

lantern Duplicate No. B2 189

lantern

Ink

$\mathrm{RC}$

Packet

number number number size

$\mathrm{RC} 817$

RC818

$\mathrm{RC} 819$

RC820

RC821

$\mathrm{RC} 822$

$\mathrm{RC} 823$

$\mathrm{RC} 824$

$\mathrm{RC} 825$

$\mathrm{RC} 826$

$\mathrm{RC} 827$

RC828

$\mathrm{RC} 829$

$\mathrm{RC} 830$

RC831

$\mathrm{RC} 832$

$\mathrm{RC} 833$

RC834

$\mathrm{RC} 835$

$\mathrm{RC} 836$

$\mathrm{RC} 837$

$\mathrm{RC} 838$

RC839

RC840

RC841

RC842

RC843 


\section{$\mathrm{RC} 844$ \\ $\mathrm{RC} 845$ \\ RC846 \\ RC847 \\ RC848 \\ RC849 \\ RC850 \\ RC851 \\ RC852 \\ RC853 \\ $\mathrm{RC} 854$ \\ $\mathrm{RC} 855$ \\ $\mathrm{RC} 856$ \\ $\mathrm{RC} 857$ \\ RC858 \\ RC859 \\ RC860 \\ RC861 \\ RC862 \\ RC863 \\ RC864 \\ RC865 \\ RC866 \\ RC867 \\ RC868 \\ RC869 \\ RC870 \\ RC871}

section section section B3 220 section section section section section section section section section B3 201 section section section section section section section section section B3 210 B2 20O B2 198 B2 189 B2 197 B2 196 B2 195 B2 194 B2 193 B2 191 B2 198 B2 190 B2 189 B2 186 B2 187 B2 188 B2 185 B2 184 B2 183 B2 192 B2 180 B2 170 B2 179 B2 178 B2 177 B2 176 B2 175 B2 174 B2 173 B2 172 B2 171 B2 169

presumed presumed presumed

657 presumed presumed presumed presumed presumed presumed presumed presumed presumed

656 presumed presumed presumed presumed presumed presumed presumed presumed presumed

652

641

650

649

648

647 
Packet

Ink

Slide Comments

number number number size $^{4}$

RC872

B2

145

599

lantern

RC873

B2 168 
RC874

B2

167

621

RC875

B2

166

620

RC876

B2

165

619

RC877

B2 164

618

RC878

B2

163

617

RC879

B2

162

616

RC880 
RC883

B2

158

612

RC884

B2

157

611

RC885

B2

156

609

B2 156

RC886

B2

155

608

lantern

lantern Duplicate No. 
RC887

B2 154

607

RC888

B2

153

606

RC889

B2 156

610

RC890

B2

151

605

RC891

B2

150

604

RC892

B2

149

603

RC893

B2 148

602 
RC894

B2

147

601

RC895

B2

146

600

RC896

B2

135

589

lantern

RC897

B2

144

598

RC898

B2

143

597

RC899

B2

142

596

RC900

B2

141 
595

RC901

B2

140

594

RC902

B2

139

593

RC903

B2

138

592

RC904

B2

137

591

RC905

B2

136

590

RC906

B2

120

583

lantern 
RC907

B2

134

588

RC908

B2

133

587

RC909

B2

132

586

RC 910

B2

131

585

RC911

B2

130

584

RC 912

B1 29

509

RC913

B1 28

508 
RC914

B1 27

507

RC915

B1 26

506

RC916

B1 25

505

RC917

B1

24

504

RC918

B1

23

503

RC919

Bl

22

502

RC 920

B1 21

501 
RC921

B1

199

953

RC922

B1

18

498

RC923

B1

17

497

RC924

B1

16

496

RC925

B1

15

495

RC926

B1

14

494 


\section{Appendix}

00000000000000000000000000000000000000000000

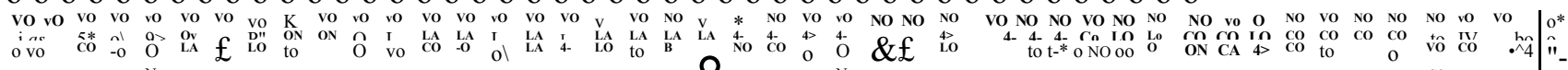

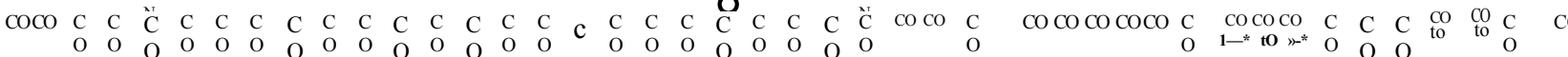

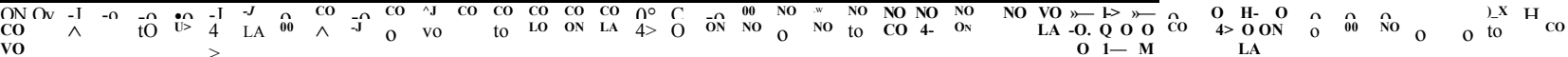
LA

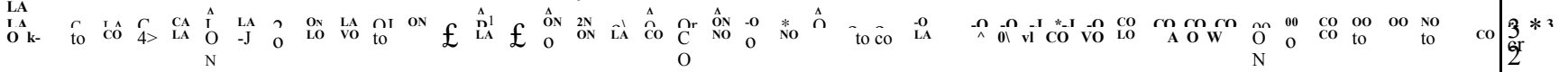

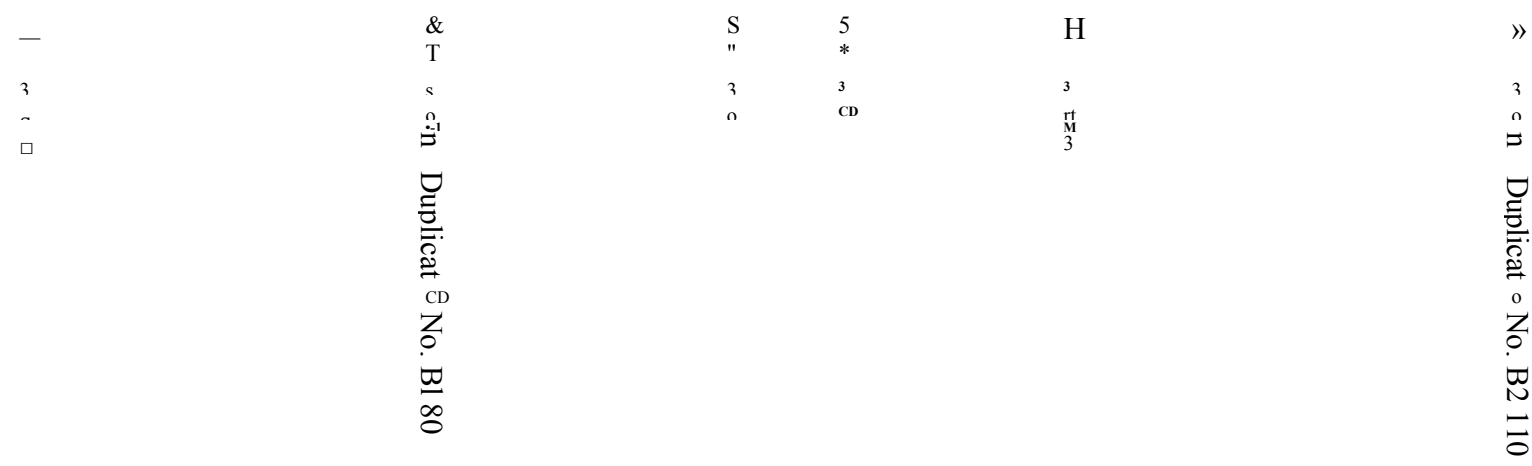

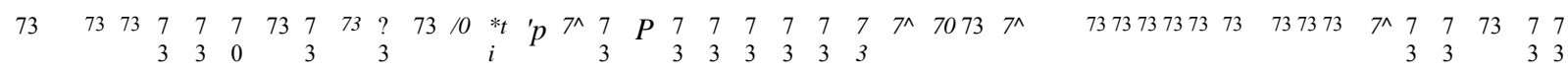

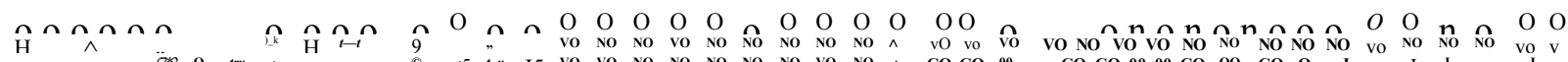

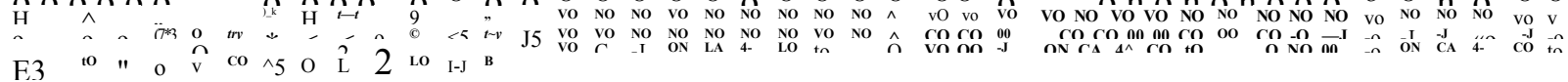

$\begin{array}{llllllllllllllllll} & \mathrm{C} & \mathrm{C} & \mathrm{C} & \mathrm{C} & \mathrm{C} & \mathrm{C} & \mathrm{C} & \mathrm{C} & \mathrm{C} & \mathrm{C} & \mathrm{C} & \mathrm{C} & \mathrm{CO} & \mathrm{CO} & \mathrm{c}\end{array}$

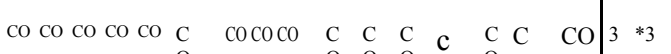

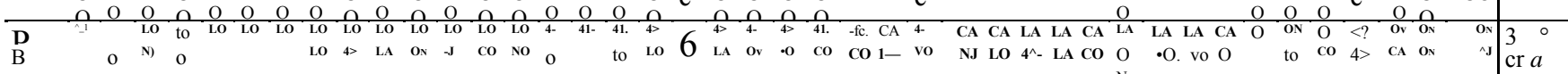

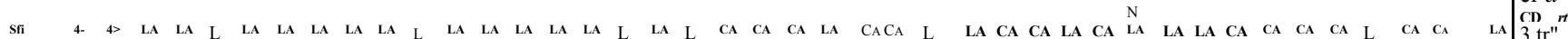

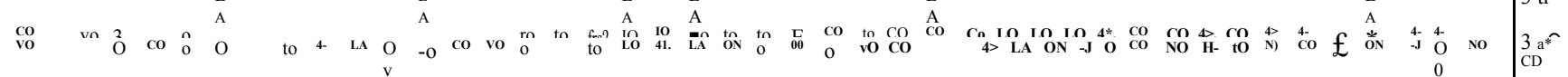
$\stackrel{\mathrm{D}}{\stackrel{2}{\rightleftarrows}} \stackrel{2}{\mathrm{n}} \stackrel{3}{\mathrm{n}}$

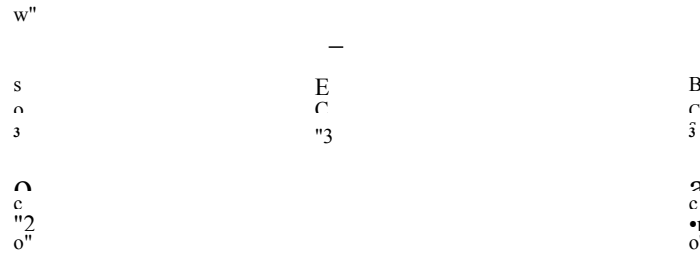

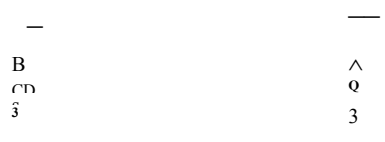

c
$\bullet n$
$o^{\prime}$ 
